\title{
Continuous-wave Optically Pumped Lasing of Hybrid Perovskite VCSEL at Green Wavelength
}

\author{
Mohd Sharizal Alias ${ }^{1}$, Zhixiong Liu ${ }^{2}$, Abdullah Al-Atawi ${ }^{1}$, Tien Khee $\mathrm{Ng}^{1}$, Tom $\mathrm{Wu}^{2}$, and Boon S. Ooi ${ }^{1 \text {,* }}$ \\ ${ }^{1}$ King Abdullah University of Science and Technology (KAUST), Photonics Laboratory, Computer, Electrical and Mathematical Science and \\ Engineering Division (CEMSE), Thuwal 23955-6900, Saudi Arabia \\ ${ }^{2}$ King Abdullah University of Science and Technology (KAUST), Materials Science Engineering, Division of Physical Science and Engineering \\ (PSE), Thuwal 23955-6900, Saudi Arabia \\ Authore-mail address: boon.ooi@kaust.edu.sa
}

\begin{abstract}
We demonstrate the lasing of a perovskite vertical-cavity surface-emitting laser at green wavelengths, which operates under continuous-wave optical pumping at room-temperature by embedding hybrid perovskite between dielectric mirrors deposited at low-temperature.

OCIS codes: (160.3380) Laser materials; (140.7260) Vertical cavity surface emitting lasers; (140.7300) Visible lasers.
\end{abstract}

\section{Introduction}

Hybrid perovskites are attracting research interest for light-emitting devices (LEDs and lasers) because they can demonstrate high luminescence, high carrier mobility, tunable visible emission, and low-cost solution processing [1, 2]. These characteristics suggest hybrid perovskites could address the green lasing spectrum gap (520-550 nm), in addition to the existing approaches of using group III-nitride- and organic-based materials.

Several hybrid perovskites vertical-cavity surface-emitting laser (Pe-VCSELs) have been reported recently, however, most of the emission wavelengths were at near-infrared spectrum [3-5]. Only one Pe-VCSEL that was fabricated using the cast-capping method was shown to exhibit green spectrum emission [6]. Although an optical pumping threshold was observed, only amplified spontaneous emission (ASE) is acquired due to its higher threshold $(>2 \times)$ and broader linewidth $(>3 \times)$ when compared to an edge-emitting laser prepared using the same method. The high pumping threshold is expected because of the poor bonding quality formed between the casted interfaces and the thick hybrid perovskite crystals used (around 3.6 $\mu \mathrm{m}$ ). Furthermore, the non-uniformity of the crystal thickness and surface flatness caused by the cast-capping method resulted in fluctuation of the cavity thickness. This contributed to the deviation of the emission wavelength (up to $39 \mathrm{~nm}$ ) from the initial emission. So far, all of the reported Pe-VCSELs have been optically pumped using high-power nano- to femto-second laser pulses. Achieving lasing using continuous wave $(\mathrm{CW})$ optical pumping would be an important step towards realizing a $\mathrm{CW}$ electrically injected hybrid perovskites laser. Here we demonstrate green lasing of a Pe-VCSEL under CW optical pumping at room-temperature by embedding a hybrid perovskite heterostructure-type active region of cavity lambda thickness within well-bonded dielectric Distributed Bragg Reflectors (DBRs) deposited at a low-temperature.
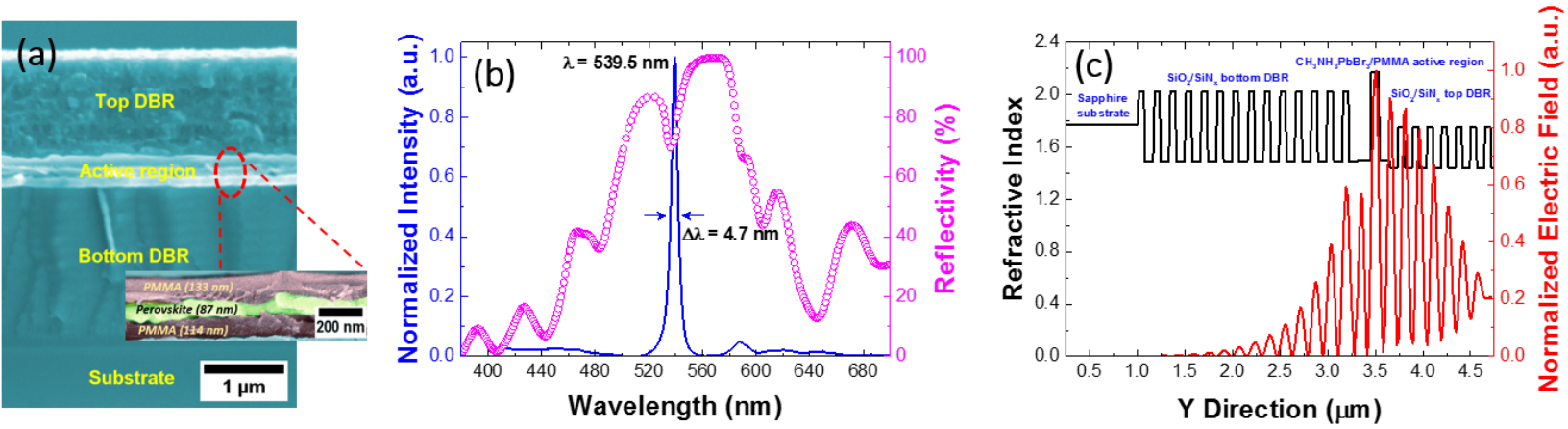

Fig. 1. (a) Cross-sectional micrograph of the Pe-VCSEL. (b) Room-temperature PL and reflectivity spectra of the Pe-VCSEL at the highest pumping excitation $\left(664 \mathrm{~kW} / \mathrm{cm}^{2}\right)$. (c) The refractive index profile and optical standing wave across the Pe-VCSEL.

\section{Experiments and results}

Figure 1a shows the cross-sectional micrograph of the Pe-VCSEL composed of a bottom dielectric DBR deposited at standard temperature $\left(300^{\circ} \mathrm{C}\right)$ on a sapphire substrate, an active region containing the $\mathrm{CH}_{3} \mathrm{NH}_{3} \mathrm{PbBr}_{3}$ perovskite, and a top dielectric DBR deposited at low-temperature $\left(60^{\circ} \mathrm{C}\right)$. We embedded the $\mathrm{CH}_{3} \mathrm{NH}_{3} \mathrm{PbBr}_{3}$ thin film between poly(methyl methacrylate) (PMMA) spacers (inset Fig. 1a) to acquire heterostructured active region with a cavity lambda thickness. The $\mathrm{CH}_{3} \mathrm{NH}_{3} \mathrm{PbBr}_{3}$ thin film was deposited using the method of sequential vapor deposition and 
vapor annealing, whereas the dielectric DBRs were deposited using plasma-enhanced chemical vapor deposition. In Fig. 1b, we observed a clear dip in reflectivity with a narrow spectral width originating from a resonant transmission through the DBR due to the cavity mode. This shows that a resonance vertical microcavity structure has been formed. The cavity $Q$-factor was estimated from the ratio of cavity emission wavelength with the linewidth $(\lambda \Delta \lambda)$ which is about 115 . We also optimally designed the hybrid perovskite layer at the antinode of the standing wave (Fig. 1c) to ensure low-threshold lasing. The standing wave profile was simulated using a transfer-matrix method solver based on measured refractive index and thickness of each layer in the Pe-VCSEL.

Room-temperature photoluminescence (PL) spectra were acquired using a micro-PL spectrometer with a $325 \mathrm{~nm}$ excitation CW laser. Fig. 2a shows the pump-dependent emission of the Pe-VCSEL using a range of CW excitation pump densities. We observed that the emission wavelength red-shifted as well as significantly intensified and narrowed with an increment of pumping density, indicating the evolution from ASE to lasing. Above the threshold, uniform and continuous green emission were observed at the near-field profile of the Pe-VCSEL (inset Fig. 2a). We compared the emissions from the Pe-VCSEL and pristine hybrid perovskite sample at the highest pumping excitation (Fig. 2 b) and found that emission was $\sim 7 \times$ more intense and $\sim 0.18 \times$ narrower for the Pe-VCSEL.
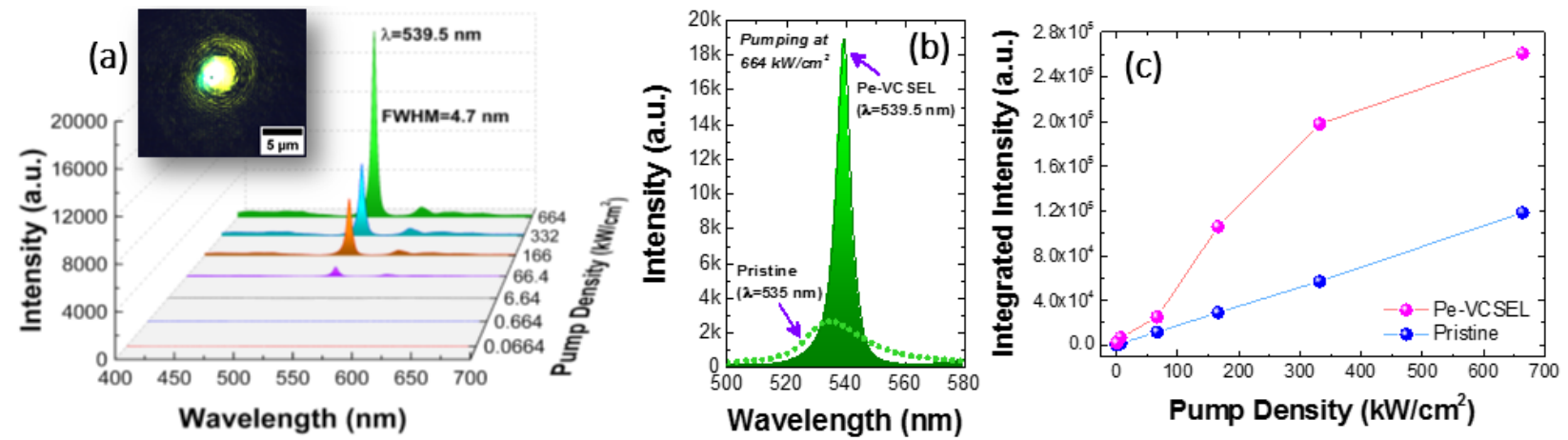

Fig. 2. (a) Emission spectra of the Pe-VCSEL at various pumping excitations with inset displaying the near-field profile. (b) Comparison of the room-temperature PL spectra between the Pe-VCSEL and the pristine hybrid perovskite sample at the highest pumping excitation $\left(664 \mathrm{~kW} / \mathrm{cm}^{2}\right)$.

(c) The plot of the integrated room-temperature PL intensity vs. pumping density for the Pe-VCSEL and pristine hybrid perovskite sample.

Figure 2(c) shows the integrated intensity of the PL spectra as a function of pumping density for the Pe-VCSEL and the pristine hybrid perovskite sample. We observed lasing threshold for the Pe-VCSEL at a pumping density of $\sim 66.4 \mathrm{~kW} / \mathrm{cm}^{2}$. The slope efficiency increased from $175.6 \mathrm{~kW}^{-1} \mathrm{~cm}^{-2}$ (for the pristine hybrid perovskite sample) to $812.5 \mathrm{~kW}^{-1} \mathrm{~cm}^{-2}$ (for the Pe-VCSEL). We observed roll-over (self-heating effect) in the lasing characteristics of our Pe-VCSEL beyond a pumping density of $170 \mathrm{~kW} / \mathrm{cm}^{2}$. While the Pe-VCSEL lased at higher pumping densities, the pristine hybrid perovskite sample only demonstrated plateau ASE characteristic.

\section{Conclusions}

In summary, we have demonstrates room temperature CW optically pumped Pe-VCSEL that can lase at green wavelengths. This achievement was enabled with a design that incorporated a thin heterostructured hybrid perovskites active region of a cavity lambda thickness and a well-bonded dielectric DBR top-layer that was deposited at low-temperature. We demonstrate that our Pe-VCSEL can lase continuously without high-power laser pulsed pumping. With further improvements to the device configuration, such as forming a mesa and using proper electron-hole transport layers, the Pe-VCSEL can be made for $\mathrm{CW}$ electrical injection using standard microfabrication processes, thus offering a promising route towards realizing a true green laser.

\section{References}

[1] S. A. Veldhuis, P. P. Boix, N. Yantara, et al., "Perovskite materials for light-emitting diodes and lasers," Adv. Mater. 28, 6804 (2016).

[2] David B. R. Sutherland and E. H. Sargent, "Perovskite photonic sources," Nat. Photonics 10, 295 (2016).

[3] F. Deschler, M. Price, S. Pathak, et al., "High photoluminescence efficiency and optically pumped lasing in solution-processed mixed halide perovskite semiconductors," J. Phys. Chem. Lett. 5, 1421 (2014).

[4] S. D. Stranks, S. M. Wood, K. Wojciechowski, et al., "Enhanced amplified spontaneous emission in perovskites using a flexible cholesteric liquid crystal reflector," Nano Lett. 15, 4935 (2015).

[5] S. Chen, C. Zhang, J. Lee, et al., "Hybrid perovskite vertical-cavity surface-emitting laser deploying nanoporous GaN dielectric reflector technology," in Conference on Lasers and Electro-Optics, OSA Technical Digest (2016) (Optical Society of America, 2016), SW1M.2.

[6] V.-C. Nguyen, H. Katsuki, F. Sasaki, et al., "Optically pumped lasing in single crystals of organometal halide perovskites prepared by castcapping method," Appl. Phys. Lett. 108, 261105 (2016). 